\title{
Time-odd mean fields in the rotating frame: microscopic nature of nuclear magnetism.
}

\author{
A. V. Afanasjert and P. Ring \\ Physik-Department der Technischen Universität München, D-85747 Garching, Germany
}

(October 26, 2018)

\begin{abstract}
The microscopic role of nuclear magnetism in rotating frame is investigated for the first time in the framework of the cranked relativistic mean field theory. It is shown that nuclear magnetism modifies the expectation values of singleparticle spin, orbital and total angular momenta along the rotational axis effectively creating additional angular momentum. This effect leads to the increase of kinematic and dynamic moments of inertia at given rotational frequency and has an impact on effective alignments.
\end{abstract}

PACS numbers: 21.60.-n, 21.60.Cs, 21.60.Jz 
It is a well established fact that microscopic mean field theories provide a good description of the properties of finite nuclei. The most succesful among them are the non-relativistic theories based on the finite range forces of the Gogny type and zero-range forces of the Skyrme type [自] and relativistic mean field (RMF) theory [2]. In these theories the physical observables are most sensitive to the time-even fields, which has widely been investigated throughout the periodic table. On the other hand, the properties of time-odd fields, which appear only in the nuclear systems with broken time-reversal symmetry, are poorly known. However, it is known nowadays that these fields are very important for the proper description of rotating nuclei [3] [ magnetic moments [7], the properties of the $N=Z$ nuclei [8] and pairing correlations [9].

In rotating nuclei, the time-odd mean fields emerge both from the Coriolis operator and from the parts of the Hamiltonian (Lagrangian) related to the currents. Both in relativistic [4,6] and non-relativistic [3,5, 10] mean field theories the most important modification coming from the presence of currents lies in the renormalization of the moments of inertia. They increase the kinematic $\left(J^{(1)}\right)$ and dynamic $\left(J^{(2)}\right)$ moments of inertia by $\sim 20-30 \%$. However, the microscopic mechanism of these modifications has not been well understood till now.

The aim of the present article is to investigate this mechanism staying strictly in the framework of the cranking model. As a theoretical tool we are using the cranked relativistic mean field (CRMF) theory [11, [4,6] in which the time-odd mean fields are defined in a unique way. Since we are interested in a general understanding of the microscopic mechanism of the renormalization of the moments of inertia, the pairing correlations are neglected in the present investigation.

In RMF theory the nucleus is described as a system of point-like nucleons (Dirac spinors) coupled to the mesons and to the photons. The nucleons interact by the exchange of several mesons: isoscalar scalar $\sigma$-meson, isoscalar vector $\omega$-meson, isovector vector $\rho$-meson and the photon. The transformation to the rotating frame in the one-dimensional cranking approximation leads to the CRMF theory, the details of which are given in Refs. [11, 12, 田, 6]. The main features of this theory important in the present discussion are outlined below. 
In the Hartree approximation, the stationary Dirac equation for the nucleons in the rotating frame is given by

$$
\begin{array}{r}
\left\{\boldsymbol{\alpha}(-i \boldsymbol{\nabla}-\boldsymbol{V}(\boldsymbol{r}))+V_{0}(\boldsymbol{r})+\beta(m+S(\boldsymbol{r}))-\Omega_{x} \hat{J}_{x}\right\} \\
\psi_{i}= \\
=\epsilon_{i} \psi_{i}
\end{array}
$$

where $V_{0}(\mathbf{r})$ represents a repulsive vector potential:

$$
V_{0}(\boldsymbol{r})=g_{\omega} \omega_{0}(\boldsymbol{r})+g_{\rho} \tau_{3} \rho_{0}(\boldsymbol{r})+e \frac{1-\tau_{3}}{2} A_{0}(\boldsymbol{r})
$$

which contains time-like components of the vector mesons and $S(\mathbf{r})$ an attractive scalar potential:

$$
S(\boldsymbol{r})=g_{\sigma} \sigma(\boldsymbol{r})
$$

A magnetic potential $\boldsymbol{V}(\boldsymbol{r})$ :

$$
\boldsymbol{V}(\boldsymbol{r})=g_{\omega} \boldsymbol{\omega}(\boldsymbol{r})+g_{\rho} \tau_{3} \boldsymbol{\rho}(\boldsymbol{r})+e \frac{1-\tau_{3}}{2} \boldsymbol{A}(\boldsymbol{r})
$$

originates from the space-like components of the vector mesons. Note that in these equations, the four-vector components of the vector fields $\omega^{\mu}, \rho^{\mu}$ and $A^{\mu}$ are separated into the time-like $\left(\omega_{0}, \rho_{0}\right.$ and $\left.A_{0}\right)$ and space-like $\left[\boldsymbol{\omega} \equiv\left(\omega^{x}, \omega^{y}, \omega^{z}\right), \boldsymbol{\rho} \equiv\left(\rho^{x}, \rho^{y}, \rho^{z}\right)\right.$, and $\left.\boldsymbol{A} \equiv\left(A^{x}, A^{y}, A^{z}\right)\right]$ components. Finally the term

$$
-\Omega_{x} \hat{J}_{x}=-\Omega_{x}\left(\hat{L}_{x}+\frac{1}{2} \hat{\Sigma}_{x}\right)
$$

represents the Coriolis field.

The time-independent inhomogeneous Klein-Gordon equations for the mesonic fields are given by

$$
\begin{gathered}
\left\{-\Delta-\left(\Omega_{x} \hat{L}_{x}\right)^{2}+m_{\sigma}^{2}\right\} \sigma(\boldsymbol{r})=-g_{\sigma}\left[\rho_{s}^{p}(\boldsymbol{r})+\rho_{s}^{n}(\boldsymbol{r})\right] \\
-g_{2} \sigma^{2}(\boldsymbol{r})-g_{3} \sigma^{3}(\boldsymbol{r}), \\
\left\{-\Delta-\left(\Omega_{x} \hat{L}_{x}\right)^{2}+m_{\omega}^{2}\right\} \omega_{0}(\boldsymbol{r})=g_{\omega}\left[\rho_{v}^{p}(\boldsymbol{r})+\rho_{v}^{n}(\boldsymbol{r})\right], \\
\left\{-\Delta-\left(\Omega_{x}\left(\hat{L}_{x}+\hat{S}_{x}\right)\right)^{2}+m_{\omega}^{2}\right\} \boldsymbol{\omega}(\boldsymbol{r})=g_{\omega}\left[\boldsymbol{j}^{p}(\boldsymbol{r})+\boldsymbol{j}^{n}(\boldsymbol{r})\right],
\end{gathered}
$$


with source terms involving the various nucleonic densities and currents

$$
\begin{aligned}
\rho_{s}^{n, p}(\boldsymbol{r}) & =\sum_{i=1}^{N, Z}\left(\psi_{i}(\boldsymbol{r})\right)^{\dagger} \hat{\beta} \psi_{i}(\boldsymbol{r}), \\
\rho_{v}^{n, p}(\boldsymbol{r}) & =\sum_{i=1}^{N, Z}\left(\psi_{i}(\boldsymbol{r})\right)^{\dagger} \psi_{i}(\boldsymbol{r}), \\
\boldsymbol{j}^{n, p}(\boldsymbol{r}) & =\sum_{i=1}^{N, Z}\left(\psi_{i}(\boldsymbol{r})\right)^{\dagger} \hat{\boldsymbol{\alpha}} \psi_{i}(\boldsymbol{r}),
\end{aligned}
$$

where the labels $n$ and $p$ are used for neutrons and protons, respectively. In the equations above, the sums run over the occupied positive-energy shell model states only (no-sea approximation) [13]. For simplicity, the equations for the $\rho$ meson and the Coulomb fields are omitted in Eqs. (6) since they have the structure similar to the equations for the $\omega$ meson. Since the coupling constant of the electromagnetic interaction is small compared with the coupling constants of the meson fields, the Coriolis term for the Coulomb potential $A_{0}(\boldsymbol{r})$ and the spatial components of the vector potential $\boldsymbol{A}(\boldsymbol{r})$ are neglected in the calculations. Note that in the CRMF theory the currents $\boldsymbol{j}^{n, p}(\boldsymbol{r})$ are the products of the large $(G)$ and small $(F)$ components of the Dirac spinors $\psi_{i}(\boldsymbol{r})$.

The Coriolis operator $\hat{J}_{x}$ and the magnetic potential $\boldsymbol{V}(\boldsymbol{r})$ in the Dirac equation as well as the currents $\boldsymbol{j}^{n, p}(\boldsymbol{r})$ in the Klein-Gordon equations do not appear in the RMF equations for time-reversal systems [14] since they break this symmetry [15]. Similar to the nonrelativistic case [1], the presence of the Coriolis operator leads to the appearance of the time-odd mean fields. However, the CRMF calculations with only these time-odd fields accounted underestimate the experimental moments of inertia by 20-30\% [4.6]. A similar situation holds also in non-relativistic theories, see Refs. [0,10] and references therein. The inclusion of the currents $\boldsymbol{j}^{n, p}(\boldsymbol{r})$, which leads to the space-like components of the vector $\omega$ and $\rho$ mesons and thus to magnetic potential $\boldsymbol{V}(\boldsymbol{r})$, considerably improves the description of experimental moments of inertia. The effect coming from the space-like components of the vector mesons is commonly referred as nuclear magnetism (further NM) [11] in the framework of RMF theory.

Before we discuss the microscopic impact of NM on the moments of inertia, we remind 
that the kinematic $\left(J^{(1)}\right)$ and dynamic $\left(J^{(2)}\right)$ moments of inertia are defined as

$$
J^{(1)}\left(\Omega_{x}\right)=\frac{J}{\Omega_{x}}, \quad J^{(2)}\left(\Omega_{x}\right)=\frac{d J}{d \Omega_{x}}
$$

where the rotational frequency $\Omega_{x}$ along the $x$-axis in a one-dimensional cranking approximation is determined by the condition that the expectation value of the total angular momentum $J$ at spin $I$ has a definite value [16]:

$$
J=\left\langle\Phi_{\Omega}\left|\hat{J}_{x}\right| \Phi_{\Omega}\right\rangle=\sqrt{I(I+1)}=\sum_{i=1}^{A}\left\langle i\left|\hat{j}_{x}\right| i\right\rangle
$$

Note that $J$ is defined in the cranking model as a sum of the expectation values of the single-particle angular momentum operators $\hat{j}_{x}$ of the occupied states. This suggests that the modifications of the moments of inertia due to NM should be traced to the changes of the single-particle $\left\langle\hat{j}_{x}\right\rangle_{i}$ values.

In order to confirm this expectation the CRMF calculations with and without NM (the later will be further denoted as WNM) have been performed for the doubly magic superdeformed (SD) configuration in ${ }^{152} \mathrm{Dy}\left(\pi 6^{4} \nu 7^{2}\right)$ [6]. This configuration has been selected due to the large stability of the SD minimum against rotation. The calculated $\left\langle\hat{j}_{x}\right\rangle_{i}$ values of the single-neutron orbitals forming the $N=1$ shell are given in Fig. 1. This shell has been selected because (i) it is reasonably well separated in energy from the $N=3$ shell and thus the emerging picture is not strongly disturbed by an interaction between the $N=1$ and the $N=3$ shells, (ii) the number of single-particle orbitals is, from one hand, reasonably small to make an analysis transparent and, from other hand, sufficient to make meaningful conclusions.

Indeed NM has a considerable impact on the expectation values of the single-particle angular momentum $\left\langle\hat{j}_{x}\right\rangle_{i}$. If we define the contribution to $\left\langle\hat{j}_{x}\right\rangle_{i}$ due to NM as

$$
\Delta\left\langle j_{x}\right\rangle_{i}=\left\langle\hat{j}_{x}\right\rangle_{i}^{N M}-\left\langle\hat{j}_{x}\right\rangle_{i}^{W N M}
$$

then one can see in Fig. 1 that $\Delta\left\langle j_{x}\right\rangle_{i}$ is positive at the bottom and negative at the top of the $N=1$ shell. The absolute value of $\Delta\left\langle j_{x}\right)$ correlates with the absolute value of $\left\langle\hat{j}_{x}\right\rangle_{i}$. 
Note that the contributions to $\left\langle\hat{j}_{x}\right\rangle_{i}$ due to NM are small in the middle of the shell. It was checked also that similar features hold also in higher- $N$ shells. Note that the total angular momentum built within the $N=1$ shell is not equal 0 (see top panel in Fig. 1) due to the admixture of the $N=3$ shell and that NM increases mixing of the $N=3$ and $N=1$ shells as seen from an increased value of this momentum and from the analysis of the structure of the wave functions of the single-particle orbitals. These results suggest that if it would be possible to isolate the $N$-shell then the contribution of NM to total angular momentum of this shell would be equal zero.

In order to gain a deeper understanding of the impact of NM, the angular momentum of the particle along the rotation axis is separated into the orbital and the spin parts: $\hat{j}_{x}=\hat{l}_{x}+\hat{s}_{x}$. Then the contributions due to NM to the expectation values of the orbital $\left(\Delta\left\langle\hat{l}_{x}\right\rangle_{i}\right)$ and the spin $\left(\Delta\left\langle\hat{s}_{x}\right\rangle_{i}\right)$ angular momenta, which are defined similar to Eq. (10), are plotted in Fig. 2 .

The spin angular momenta of the particles in the $[110] 1 / 2^{+}$and $[110] 1 / 2^{-}$orbitals, which are the lowest orbitals in the $N=1$ shell, are almost fully aligned along the axis of rotation already at $\Omega_{x}=0.0 \mathrm{MeV}$. Their expectation values $\left\langle\hat{s}_{x}\right\rangle_{i}$ are equal to $\sim 0.49 \hbar$ and $\sim-0.49 \hbar$ at all rotational frequencies employed. Thus NM cannot have an impact on the spin angular momenta of the particles in these orbitals $\left(\Delta\left\langle\hat{s}_{x}\right\rangle_{i} \approx 0 \hbar\right.$, see Figs. 2a,b) and the contributions to $\left\langle\hat{j}_{x}\right\rangle_{i}$ due to NM are almost solely defined by the modifications of the expectation values of the orbital angular momentum $\left\langle\hat{l}_{x}\right\rangle_{i}$ induced by NM, see Figs. 2a,b. On the contrary, the spin angular momenta of the particles in the other orbitals forming the $N=1$ shell are only partially aligned along the rotation axis. Thus NM modifies the expectation values of both the spin and orbital angular momenta of the particles in these orbitals, see Figs. 20,d,e,f. Note that the contributions $\Delta\left\langle\hat{l}_{x}\right\rangle_{i}$ and $\Delta\left\langle\hat{s}_{x}\right\rangle_{i}$ have in general different rotational frequency dependence and can have different signs. The later is clearly seen in the case of the $[101] 3 / 2^{+}$orbital, see Figs. 2c. The results for proton orbitals are very similar to the ones presented here for neutrons. Thus one can conclude that, in general, NM leads to the 
modification of alignment properties of single-particle orbitals on the level of spin, orbital and total angular momenta.

It was checked that the impact of NM on $\left\langle\hat{j}_{x}\right\rangle_{i}$ has the same general features also in other shells. However, for higher $N$-shells the crossings between the single-particle routhians make the emerging picture more complicate. The question is then how to understand the increase of kinematic and dynamic moments of inertia induced by NM. In the case of yrast SD band in ${ }^{152}$ Dy, the shells up to $N=3$ can be considered as 'closed' since all the orbitals belonging to them are occupied. The contribution of the 'closed' shells to the expectation value $J$ of the total angular momentum accounts only for few $\%$ of $J$ as illustrated on the example of the $N=1$ shell, see top panel in Fig. 1. Thus the main contribution to $J$ (and correspondingly to $J^{(1)}$ and $\left.J^{(2)}\right)$ is coming from the particles and holes outside the 'closed' shells.

In the lowest $\mathrm{SD}$ configuration of ${ }^{152} \mathrm{Dy}$, valence protons are sitting in the orbitals, emerging from the bottom of the $N=5$ and $N=6$ shells (see for example Fig. 2 in Ref. [17]), for which the contribution of NM to $\left\langle\hat{j}_{x}\right\rangle_{i}$ is positive. Valence proton holes are located in the orbitals, emerging from the middle and the top of the $N=4$ shell. The contribution of NM to $\left\langle\hat{j}_{x}\right\rangle_{i}$ for these orbitals is either close to zero or negative. However, these contributions should be taken with opposite sign due to hole nature. Thus these proton holes also give a positive contribution due to NM to the total proton angular momentum. The consideration of the neutron subsystem can be performed in a similar way and the conclusions are the same. Thus one can conclude that at given rotational frequency the modifications of single-particle $\left\langle\hat{j}_{x}\right\rangle_{i}$ values induced by NM will lead to an increase of total $J$ and, as a result, to an increase of kinematic and dynamic moments of inertia. Essentially this means that $\mathrm{NM}$ is the source of the creation of angular momentum in rotating nuclei in addition to the Coriolis force. Clearly this increase in $J, J^{(1)}$ and $J^{(2)}$ induced by NM should depend both on the rotational frequency and on the shell filling (and thus on the specific single-particle configuration).

In the light of these findings, it is important to see which other physical quantities are affected by NM. NM can have considerable impact on the signature splitting of the signature 
partner orbitals as it is clearly seen in the case of the $[110] 1 / 2^{ \pm}$and $[330] 1 / 2^{ \pm}$orbitals, see Fig. 3. One should also note that the change in $\left\langle\hat{j}_{x}\right\rangle_{i}$ induced by NM is not fully reflected in the change of the slope of the single-particle energies drawn as a function of the rotational frequency (see Fig. 3). This is contrary to the results of the phenomenological models based on the Woods-Saxon or Nilsson potentials where for a cranking at a fixed deformation, the slope of the orbital routhian against rotational frequency corresponds to the alignment $\left\langle\hat{j}_{x}\right\rangle_{i}=-\delta \epsilon_{i}^{\Omega_{x}} / \delta \Omega_{x}$, where $\epsilon_{i}^{\Omega_{x}}$ is the single-particle energy of $i$-th orbital at given $\Omega_{x}$. For example, the $[110] 1 / 2^{ \pm}$and $[101] 1 / 2^{-}$orbitals have the contributions to $\langle\hat{j}\rangle_{i}$ induced by NM (see Figs. [a $\mathrm{a}, \mathrm{b}, \mathrm{f}$ ) similar in absolute value. However, the change of the slope of the singleparticle routhians induced by $\mathrm{NM}$ is larger for the $[110] 1 / 2^{+}$orbital as compared with the $[110] 1 / 2^{+}$and $[101] 1 / 2^{-}$orbitals, see left panel of Fig. 3. This essentially suggests that the difference between the single-particle energies of the orbitals calculated with and without NM reflects not only the change of alignment properties of corresponding single-particle orbitals but also the energy gain (or cost) due to the interaction of the nucleon with the magnetic potential $\boldsymbol{V}(\boldsymbol{r})$.

In order to check that the features mentioned above are not coming from small differences in equilibrium deformations obtained in the calculations with and without NM, the calculations without NM have been performed starting from the self-consistent fields obtained in the calculations with NM and performing only one iteration during which the physical quantities of interest have been recalculated neglecting NM. Although the solution obtained in this case is not self-consistent, its equilibrium deformation differs only marginally from the one obtained in fully self-consistent calculations with NM. Using this result a similar analysis as presented above has been repeated. Although some minor quantitative differences exist, the physical conclusions are the same.

It is reasonable to expect that also the physical observables which depend on the alignment properties of specific single-particle orbitals are affected by NM. One of these observables is the effective alignment $i_{\text {eff }}$ which measures the difference between the spins 
of bands $\mathrm{A}$ and $\mathrm{B}$ at constant $\Omega_{x}: i_{\text {eff }}^{\mathrm{A}, \mathrm{B}}\left(\Omega_{x}\right)=I_{\mathrm{B}}\left(\Omega_{x}\right)-I_{\mathrm{A}}\left(\Omega_{x}\right)$ and which in CrankedNilsson+Strutinsky (CNS) approach based on the cranked Nilsson potential is predominantly defined by the alignment(s) of the single-particle orbital(s) by which the compared bands differ [18]. Fig. T shows that NM indeed can have a considerable impact (especially in the case of the $\pi[651] 3 / 2(r=+i)$ orbital $)$ on effective alignments. A similar conclusion about the impact of time-odd components of the mean field on relative (effective) alignments has been drawn earlier in the cranked Hartree-Fock approach based on Skyrme forces [5]. Note that contrary to the CNS approach polarization effects play a much more important role in the CRMF theory and thus effective alignments are not defined predominantly by single-particle alignments, see Fig. 4 . The fact that effective alignments between SD bands in different mass regions [19 21] are correctly described in the CRMF theory indicates that both alignment properties of single-particle orbitals and relevant polarization effects are properly accounted in this theory.

In conclusion, the microscopic role of nuclear magnetism in rotating frame has been investigated for the first time. The breaking of time-reversal symmetry induced by rotation results in baryonic currents which lead to the non-vanishing space-like components of the vector mesons creating the magnetic potential entering into the Dirac equation. The magnetic field created by this potential modifies the expectation values of the single-particle angular momenta effectively creating additional total angular momentum. Both the spin and the orbital single-particle angular momenta are affected by nuclear magnetism. This effect is state- and rotational frequency dependent. It leads to the increase of total angular momentum, kinematic and dynamic moments of inertia at given rotational frequency and has an impact on signature splitting, single-particle energies and on effective alignments. It is reasonable to expect that an increase in kinematic and dynamic moments of inertia resulting from the time-odd components of the mean field in the non-relativistic mean field theories [5.10] is caused by a similar microscopic mechanism as the one discussed in the present manuscript. Clearly more detailed studies of the microscopic nature of nuclear mag- 
netism both in non-rotating and rotating cases are mandatory. Such an investigation is in progress and its results will be reported later.

A.V.A. acknowledges support from the Alexander von Humboldt Foundation. This work is also supported in part by the Bundesministerium für Bildung und Forschung under the project 06 TM 979. 


\section{REFERENCES}

$\dagger \quad$ on leave of absence from the Laboratory of Radiation Physics, Institute of Solid State Physics, University of Latvia, LV 2169 Salaspils, Miera str. 31, Latvia

[1] P. Ring and P. Schuck, The Nuclear Many-body Problem (Springer Verlag, Heidelberg, 1980).

[2] P. Ring, Prog. Part. Nucl. Phys. 37, 193 (1996).

[3] U. Post, E. Wüst, and U. Mosel, Nucl. Phys. A437, 274 (1985).

[4] J. König and P. Ring, Phys. Rev. Lett. 71, 3079 (1993).

[5] J. Dobaczewski and J. Dudek, Phys. Rev. C 52, 1827 (1995).

[6] A. V. Afanasjev, J. König, and P. Ring, Nucl. Phys. A608, 107 (1996).

[7] U. Hofmann and P. Ring, Phys. Lett. B 214 (1988).

[8] W. Satuła, Proc. of Int. conf. "Nuclear Structure '98", Gatlinburg, USA, 1998, Ed. by C. Baktash, AIP 481, 114 (1999).

[9] K. Rutz, M. Bender, P.-G. Reinhard, and J. A. Maruhn, Phys. Lett. B468, 1 (1999).

[10] M. Yamagami and K. Matsuyanagi, report nucl-th/9908060

[11] W. Koepf and P. Ring, Nucl. Phys. A493, 61 (1989).

[12] W. Koepf and P. Ring, Nucl. Phys. A511, 279 (1990).

[13] P.-G. Reinhard, M. Rufa, J. Maruhn, W. Greiner, and J. Friedrich, Z. Phys. A 323, 13 (1986).

[14] B. D. Serot and J. D. Walecka, Adv. Nucl. Phys. 16, 1 (1986).

[15] W. Greiner, Relativistic quantum mechanics, Springer-Verlag, 1990

[16] D. R. Inglis, Phys. Rev. 96, 1059 (1954).

[17] T. Bengtsson and I. Ragnarsson, Nucl. Phys. A436, 14 (1985).

[18] I. Ragnarsson, Nucl. Phys. A557, 167c (1993).

[19] A. V. Afanasjev, G. A. Lalazissis, and P. Ring, Nucl. Phys. A634, 395 (1998).

[20] A. V. Afanasjev, I. Ragnarsson, and P. Ring, Phys. Rev. C 59, 3166 (1999).

[21] A. V. Afanasjev, G. A. Lalazissis, and P. Ring, Acta Phys. Hung. 6, 299 (1997). 


\section{FIGURES}

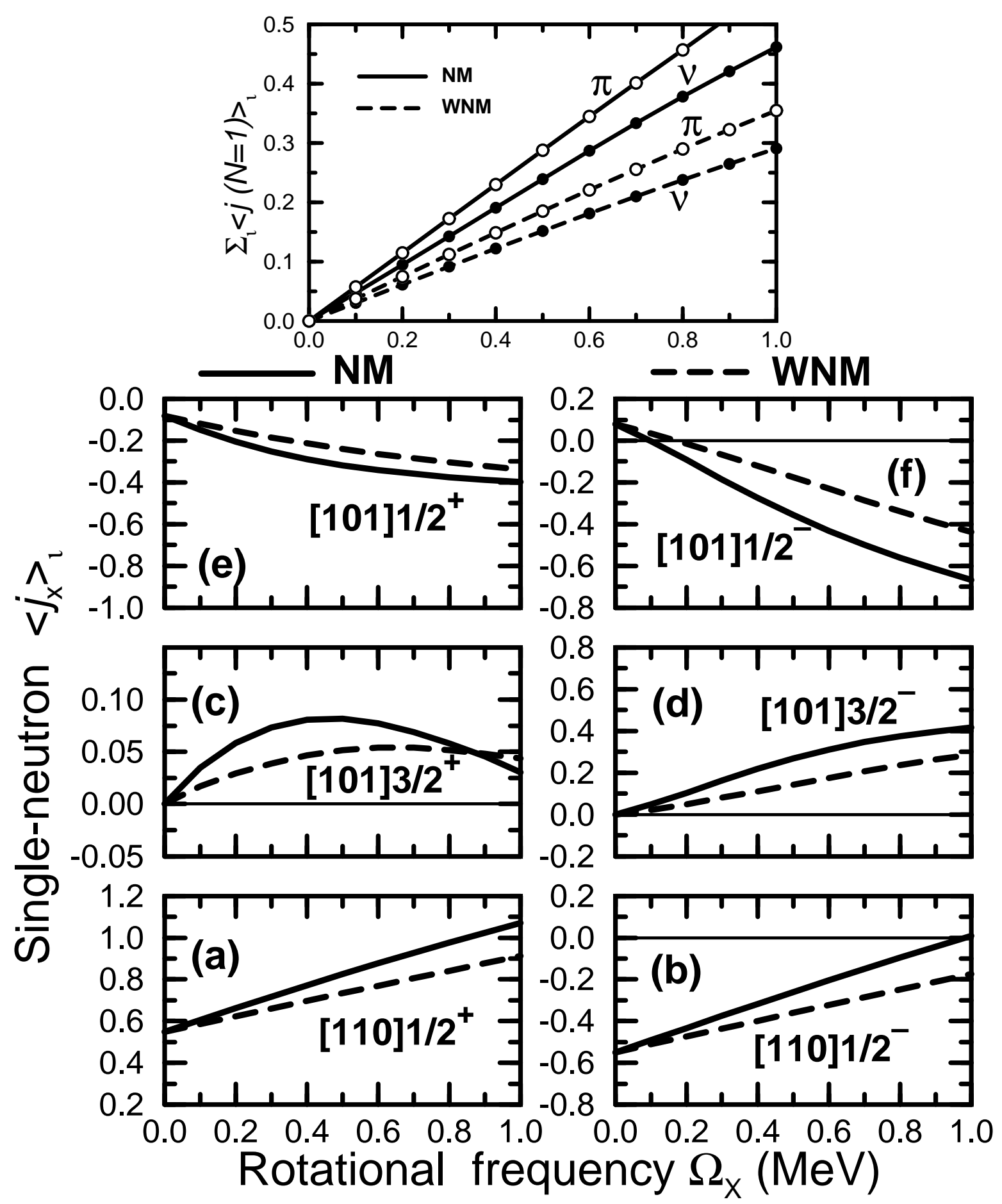


FIG. 1. The expectation values $\left\langle j_{x}\right\rangle_{i}$ of the neutron orbitals forming the $N=1$ shell calculated with and without NM. They are given along the deformation path of the lowest SD configuration in ${ }^{152} \mathrm{Dy}$. While at $\Omega_{x}=0.0 \mathrm{MeV}$ the quadrupole moments calculated with and without NM differ by $\sim 10^{-4} \%$, this difference reaches $2.7 \%$ at $\Omega_{x}=1.00 \mathrm{MeV}$. The notation of the lines is given in the figure. The single-particle orbitals are given from the bottom to the top according to their order within the $N=1$ shell, see Fig. 3. They are labeled by means of the asymptotic quantum numbers $\left[N n_{z} \Lambda\right] \Omega$ (Nilsson quantum numbers) of their dominant component of the wave function at $\Omega_{x}=0.0 \mathrm{MeV}$ and the sign of the signature quantum number $r= \pm i$ given as superscript. Since the absolute values of $\left\langle j_{x}\right\rangle_{i}$ are small for the [101]3/2 $2^{+}$orbital, the vertical scale of panel (c) is different from the other panels. In some panels, the horizontal thin lines with $\left\langle j_{x}\right\rangle=0$ are shown in order to guide eye. Top panel shows the proton and neutron angular momenta $\sum_{i}<j_{x}(N=1)>_{i}$ generated by the orbitals of the $N=1$ shell. The results of the calculations with and without $\mathrm{NM}$ are displayed. Note that in the lowest SD configuration of ${ }^{152} \mathrm{Dy}$ the total $J$ values increase smoothly from 0 at $\Omega_{x}=0.0 \mathrm{MeV}$ to $88.26 \hbar(\mathrm{NM})$ and $70.30 \hbar(\mathrm{WNM})$ at $\Omega_{x}=1.0 \mathrm{MeV}$. 


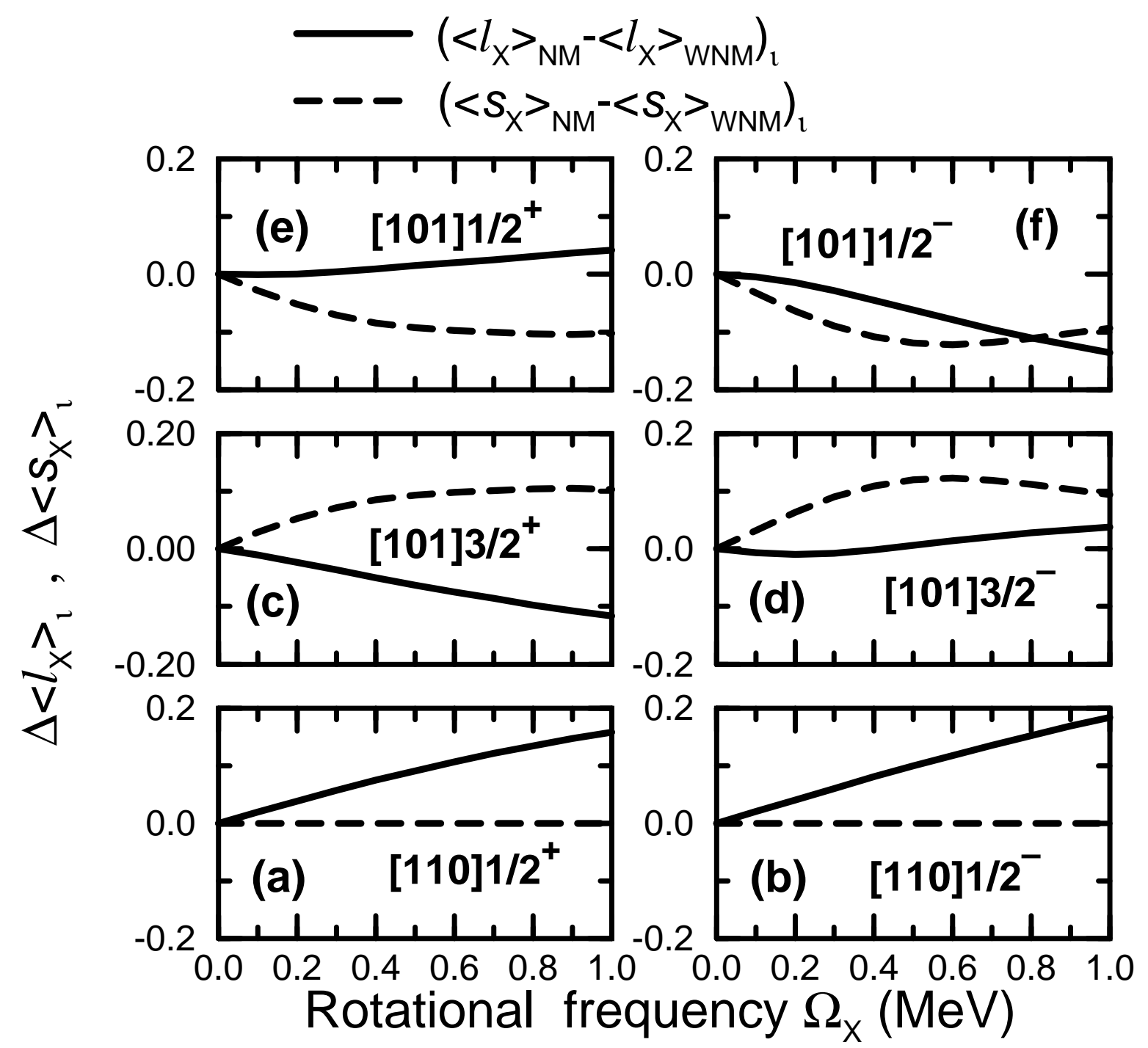

FIG. 2. The contributions to the expectation values of the spin and orbital angular momenta of the particles in the single-particle orbitals forming the $N=1$ shell caused by NM. 


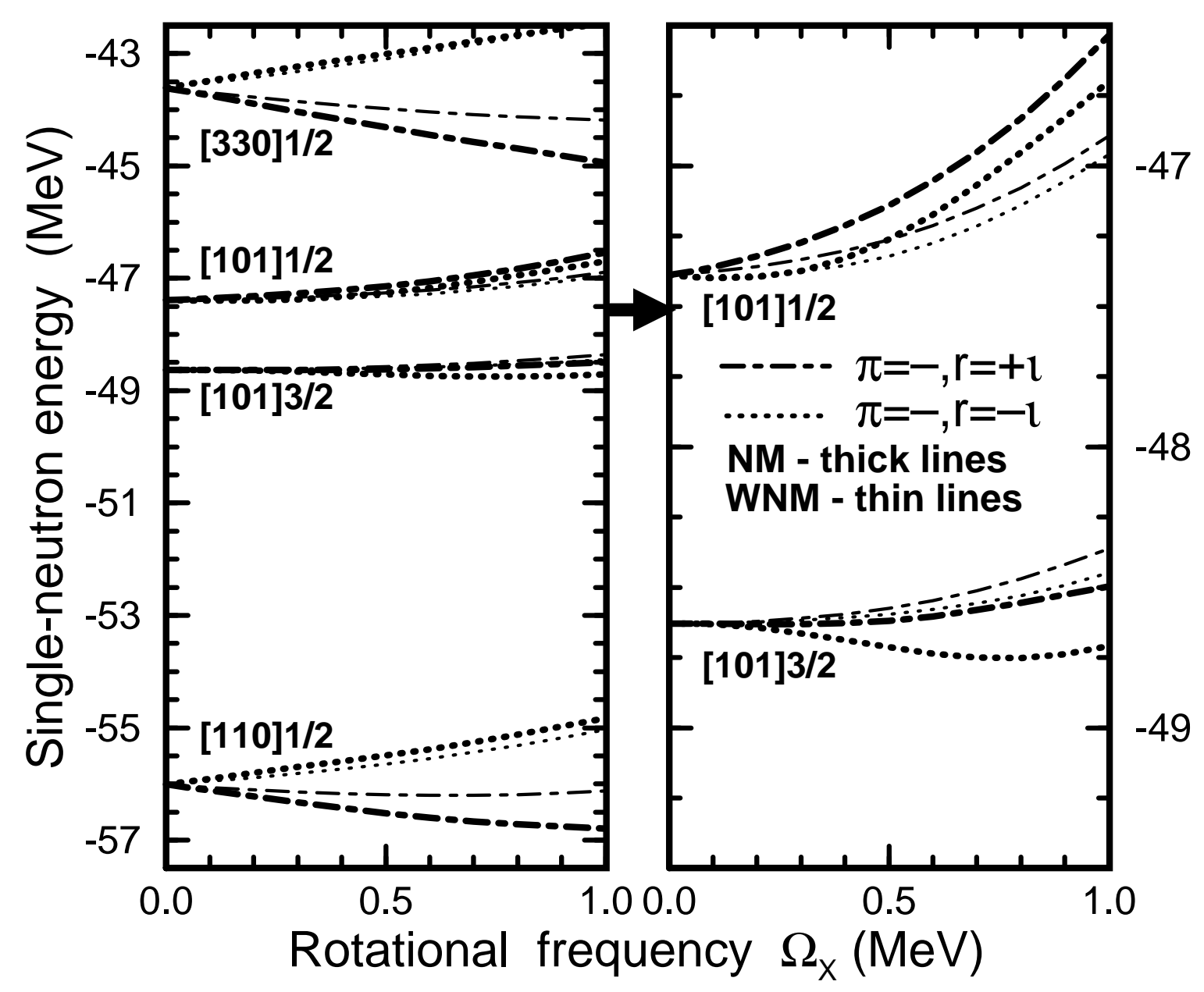

FIG. 3. Neutron single-particle energies (routhians) in the self-consistent rotating potential, calculated with and without nuclear magnetism, as a function of the rotational frequency $\Omega_{x}$. They are given along the deformation path of the lowest SD configuration in ${ }^{152} \mathrm{Dy}$. Only all the $N=1$ and the lowest $N=3$ orbitals are shown. The right panel shows the [101]3/2 and [101]1/2 orbitals in more details. The notation of the lines is given in figure. 




FIG. 4. The effective alignments (in $\hbar$ ) between the SD configurations in ${ }^{151} \mathrm{~Tb}$ and ${ }^{152} \mathrm{Dy}$ (panel (a)) and in ${ }^{152}$ Dy and ${ }^{153}$ Dy (panel (b)) are shown by the lines without symbols. The configurations in ${ }^{151} \mathrm{~Tb}$ and in ${ }^{153} \mathrm{Dy}$ are obtained with respect to the lowest $\mathrm{SD}$ configuration in ${ }^{152}$ Dy by emptying of the $\pi[651] 3 / 2(r=+i)$ orbital and by the occupation of the $\pi[402] 5 / 2(r=+i)$ orbital, see Ref. [19] for details. The expectation values of the single-particle angular momenta $\left\langle j_{x}\right\rangle_{i}$ of these orbitals are shown by the lines with symbols. Solid and open symbols are used for values calculated in ${ }^{152} \mathrm{Dy}$ and the neighboring odd nucleus, respectively. Solid and dashed lines are used for the results obtained with and without NM. In panel (b), the $\left\langle\hat{j}_{x}\right\rangle_{i}$ values calculated in ${ }^{153}$ Dy are not shown since they differ from the ones obtained in ${ }^{152}$ Dy by less than $0.026 \hbar$. 